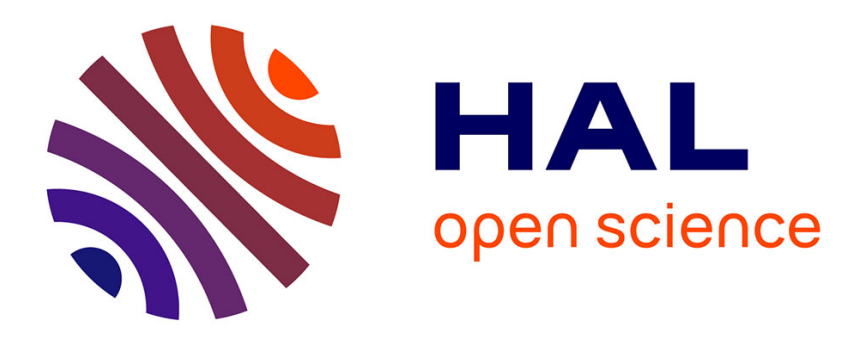

\title{
Mechanical Properties of Materials Considering Surface Effects
}

\author{
Holm Altenbach, Victor A. Eremeyev, Nikita F. Morozov
}

\section{To cite this version:}

Holm Altenbach, Victor A. Eremeyev, Nikita F. Morozov. Mechanical Properties of Materials Considering Surface Effects. 2013, pp.105-115. hal-00824006

\section{HAL Id: hal-00824006 https://hal.science/hal-00824006}

Submitted on 20 May 2013

HAL is a multi-disciplinary open access archive for the deposit and dissemination of scientific research documents, whether they are published or not. The documents may come from teaching and research institutions in France or abroad, or from public or private research centers.
L'archive ouverte pluridisciplinaire HAL, est destinée au dépôt et à la diffusion de documents scientifiques de niveau recherche, publiés ou non, émanant des établissements d'enseignement et de recherche français ou étrangers, des laboratoires publics ou privés. 


\title{
Mechanical Properties of Materials Considering Surface Effects
}

\author{
Holm Altenbach, Victor A. Eremeyev, and Nikita F. Morozov
}

\begin{abstract}
We discuss the influence of surface effects on the effective properties of materials such as the effective bending stiffness of plates or the stiffness of rods. The interest to the investigation of the surface effects is recently grown with respect to nanomechanics. The surface effects play an important role for such nanosized materials as films, nanoporous materials, etc. We consider two models of surface effects. The first one is based on the concept of surface stresses which are the generalization of the surface tension for solids. The second one (more classical approach) is based on the consideration of the thin surface layer with mechanical properties different from the bulk material. Within the framework of these models we present the effective stiffness properties of plates, shells, and nanoporous rods.

Keywords Surface stresses $\cdot$ Nanoshell $\bullet$ Nanoplate $\cdot$ Nanorod $・$ Nanoporous materials $\bullet$ Effective stiffness
\end{abstract}

\section{Introduction}

The recent progress in the nanotechnologies based on the production of new materials, so-called nanomaterials, whose properties can differ significantly from the properties of bulk materials. One of the explanations for these differences of the presence of surface effects, whose role can be extremely large for nanodimensional structures in comparison with those in classical elasticity, see [1]. Since the

\author{
H. Altenbach • V.A. Eremeyev $(\bowtie)$ \\ Otto-von-Guericke-Universität Magdeburg, Universitätplatz 2, 39106 Magdeburg, Germany \\ e-mail: holm.altenbach@ovgu.de; eremeyev.victor@gmail.com \\ N.F. Morozov \\ St. Petersburg State University, Bibliotechnaya sq. 2, 198904 St. Petersburg, Russia \\ e-mail:morozov@NM1016.spb.edu
}


contributions of Laplace, Young, and Gibbs, the mathematical study of boundaryvalue problems (BVP) for elastic bodies with surface stresses have been carried out in many works, see e.g. [1-4] and references therein. Mathematical study of BVP of linear elasticity with surface stresses is given in $[5,6]$. Recently, the theory of elasticity with surface stresses is applied to formulation of theories of nanosized structures, i.e. beams, plates, and shells, [7-17] and other surface phenomena, see e.g. $[1,18-20]$.

The aim of this work is to analyze the influence of surface effects on the elastic characteristics of materials at the nanoscale. Two models are considered. The first one is based on taking into account the surface stresses [1-4]. The surface stresses $\tau$ are the generalization of the surface tension known in the theory of capillarity for the case of solids. As is shown in [18], taking into account surface stresses results in increasing stiffness of nanoporous materials. This phenomenon is similar to increasing bending stiffness of nanoplates in comparison with the plates of macroscopic sizes $[7,8,10]$ or to increasing eigenfrequencies of bodies with surface stresses [6]. The second model uses the approach of the theory of composite materials [21-23] and the theory of laminates and sandwich plates [24, 25]. In this approach, the surface effects are taken into account due to the surface layer of finite thickness with elastic moduli differing from those of the basic material (the matrix). On the basis of these two approaches, we propose a model combining both the surface stresses and the surface layer with the properties that differ from those of the matrix.

\section{Equilibrium of Elastic Solids with Surface Stresses}

For the linear elastic body with surface stresses the boundary-value problem has the form $[1,2,4]$

$$
\begin{gathered}
\nabla \cdot \boldsymbol{\sigma}=\mathbf{0}, \quad \mathbf{x} \in V, \\
\left.\mathbf{u}\right|_{\Omega_{1}}=\mathbf{0},\left.\quad \mathbf{n} \cdot \boldsymbol{\sigma}\right|_{\Omega_{2}}=\nabla_{S} \cdot \tau+\varphi,\left.\quad \mathbf{n} \cdot \boldsymbol{\sigma}\right|_{\Omega_{3}}=\varphi, \quad \mathbf{x} \in \Omega,
\end{gathered}
$$

where $\sigma$ and $\tau$ are the stress and surface stress tensors, respectively, $\nabla$ the threedimensional nabla-operator, $\mathbf{n}$ the unit external normal to the surface $\Omega \equiv \partial V=$ $\Omega_{1} \cup \Omega_{2} \cup \Omega_{3}, V$ the volume, $\nabla_{S}$ the surface nabla-operator related with $\nabla$ by the formula $\nabla_{S}=\nabla-\mathbf{n} \partial / \partial z, z$ the coordinate along $\mathbf{n}$. The part of the body boundary $\Omega_{1}$ is clamped, on $\Omega_{2}$ the forces $\varphi$ as well as the surface tension $\tau$ are given, and the forces $\varphi$ act on $\Omega_{3}$. For isotropic materials $\sigma$ and $\tau$ are given by

$$
\boldsymbol{\sigma}=2 \mu \varepsilon+\lambda \mathbf{I t r} \varepsilon, \quad \boldsymbol{\tau}=2 \mu_{S} \mathbf{e}+\lambda_{S} \text { Atre, }
$$




$$
\begin{aligned}
\boldsymbol{\varepsilon}=\boldsymbol{\varepsilon}(\mathbf{u}) \equiv \frac{1}{2}\left(\nabla \mathbf{u}+(\nabla \mathbf{u})^{T}\right), \quad \mathbf{e} & =\mathbf{e}(\mathbf{v}) \equiv \frac{1}{2}\left(\nabla_{S} \mathbf{v} \cdot \mathbf{A}+\mathbf{A} \cdot\left(\nabla_{S} \mathbf{v}\right)^{T}\right), \\
\mathbf{v} & \left.\equiv \mathbf{u}\right|_{\Omega_{2}},
\end{aligned}
$$

where $\boldsymbol{\varepsilon}$ and $\mathbf{e}$ are the strain and surface strain tensors, respectively, $\mathbf{I}$ is the threedimensional unit tensor, $\mathbf{A}=\mathbf{I}-\mathbf{n} \otimes \mathbf{n}$ is the surface unit tensor, $\lambda, \mu$ are Lamé's moduli of the bulk material, and $\lambda_{S}, \mu_{S}$ the surface elastic moduli, which are also named the surface Lamé's moduli.

\section{Stiffness of a Rod with Surface Effects}

To illustrate the more complicated model we start from the simplest onedimensional (1D) case, i.e. from the problem of uniaxial stress state of a circular rod of radius $R$ taking into account surface effects. For the uniaxial stress state the stress tensors take the form

$$
\boldsymbol{\sigma}=\sigma_{z z} \mathbf{i}_{z} \otimes \mathbf{i}_{z}, \quad \boldsymbol{\tau}=\tau_{z z} \mathbf{i}_{z} \otimes \mathbf{i}_{z},
$$

where $\mathbf{i}_{z}$ is the unit vector along the rod axis.

We assume that the surface stresses act on the lateral surface of the rod, while the force $P$ acts at the rod ends. Equilibrium condition of the rod consists of the equation

$$
\iint_{\Omega} \sigma_{z z} d \Omega+\int_{\Gamma} \tau_{z z} d s=P
$$

where $\Omega$ is the cross-section area and $\Gamma=\partial \Omega$.

From (3) follow the 1D constitutive equations

$$
\sigma_{z z}=E \varepsilon_{z z}, \quad \tau_{z z}=E_{S} e_{z z}
$$

where $E$ and $E_{S}$ are the Young modulus of bulk material and the surface Young modulus, respectively, $\varepsilon_{z z}$ and $e_{z z}$ are the longitudinal strains. For uniaxial tension $\left.\varepsilon_{z z}\right|_{\Omega}=e_{z z}=\varepsilon$.

Assuming $\varepsilon$ to be constant and a circular cross-section of the rod with area $F=$ $\pi R^{2}$, from Eq. (6) we obtain

$$
P=\left[E F+2 \pi R E_{S}\right] \varepsilon
$$


For a homogeneous rod with the Young modulus $E^{*}$ we use the elementary formula

$$
P=E^{*} F \varepsilon
$$

Comparing (7) and (8) we obtain the effective or apparent Young's modulus of the rod with surface stresses

$$
E_{S}^{*}=E+\frac{2 E_{S}}{R} .
$$

Since $E_{S} \geq 0$, we obtain $E_{S}^{*} \geq E$. This means that the rod with surface stresses is stiffer than the same rod without surface stresses. Equation (9) predicts the sizeeffect, i.e. the dependence of the effective Young modulus on $R$, according to the scaling law [26].

Let us consider another approach to describe the surface effects. We assume that there is a thin layer of thickness $h_{\mathrm{f}}$ with the Young modulus $E_{\mathrm{f}}$. In other words we consider a non-homogeneous rod which Young modulus near the lateral surface differs from the Young modulus $E$ in the rod core.

For uniaxial tension of this non-homogeneous rod the effective Young modulus can be calculated using the mixture rule [21-23]

$$
E_{\mathrm{f}}^{*}=E+\left(E_{\mathrm{f}}-E\right) \frac{F_{\mathrm{f}}}{F},
$$

where $F_{\mathrm{f}}$ is the area of the surface layer in the rod cross-section. From (10) it follows that $E_{f}^{*} \rightarrow E_{\mathrm{f}}$ when $R \rightarrow h_{\mathrm{f}}$ and $E_{\mathrm{f}}^{*} \equiv E_{\mathrm{f}}$ when $R \leq h_{\mathrm{f}}$.

Considering both the surface stresses and the non-homogeneity of the bulk material near the lateral surface we derive the combined formula

$$
E^{*}=E+\frac{2 E_{S}}{R}+\left(E_{\mathrm{f}}-E\right) \frac{F_{\mathrm{f}}}{F} .
$$

$E^{*}$ depends on the values of $h_{\mathrm{f}}, R, E_{S}$, and $E$. The graphs of $E_{S}^{*}, E_{\mathrm{f}}^{*}$, and $E^{*}$ are presented in Fig. 1. Here $d=2 E_{S} / E$ is the characteristic length parameter introduced as in $[1,26]$. Unlike (9), Eq. (11) predicts a more complicated behavior of $E^{*}$; for example, if $E_{\mathrm{f}}<E$ then $E^{*}$ may be a non-monotonous function of $R$, see Fig. $1 b$. 
a

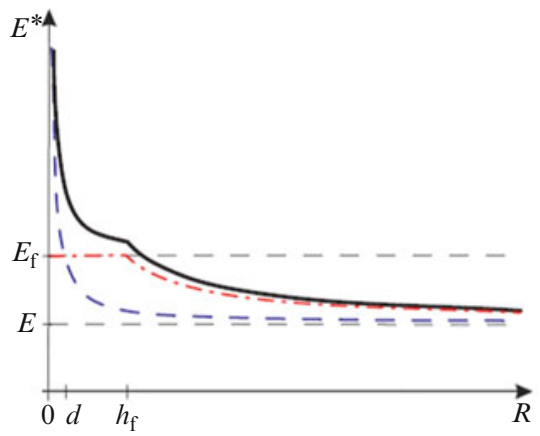

b

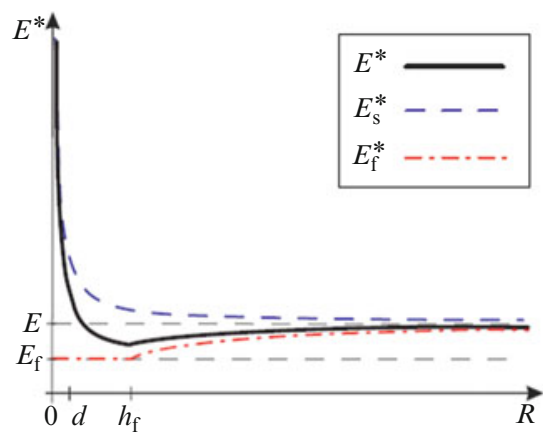

Fig. 1 Effective Young modulus $E^{*}$ vs. radius $R$ : (a) $E_{\mathrm{f}}>E$, (b) $E_{\mathrm{f}}<E$

Summarising the behavior of $E^{*}$ we can conclude that

Within the framework of the proposed model, the effective (apparent) material properties depend on the two following surface-related phenomena

- the surface elasticity according to the Gurtin-Murdoch model [2] and

- the change of the material properties of the bulk material in the vicinity of the surface.

Further we consider the application of this model to nanoporous materials and to nanosized plates and shells.

\section{Stiffness of a Nanoporous Rod}

Following [19] in this section we extend the above described procedure for the nanoporous rod. We consider a circular rod with $n$ identical pores of radius $r$ which are uniformly distributed parallel to the rod axis. We denote the area of pores in the rod cross-section as $S=\pi n r^{2}$ and introduce the porosity $\phi$ by the relation $\phi=S / F, \phi \in[0,1)$. Further, assuming the porosity is fixed we consider how the effective Young modulus depends on the number of pores.

The following formula holds true, see [19]

$$
E^{*}(n)=E(1-\phi)+E_{S} \frac{2 \sqrt{\phi F}}{\sqrt{\pi}} \sqrt{n}+\left(E_{\mathrm{f}}-E\right) \frac{F_{\mathrm{f}}(n)}{F},
$$


where $E_{\mathrm{f}}$ is the Young modulus of the bulk material in the surface layer of thickness $h_{\mathrm{f}}$ surrounding each of pore, $F_{\mathrm{f}}$ is the total area of these layers in the rod crosssection. Equation (12) shows that the porous rod can be stiffer or softer than the porous rod without the surface effects. As in Sect. 2, the surface stresses lead to the monotonous increase of $E^{*}(n)$ with the increase of $n$, while the influence of the change of the Young modulus near the pore surface depends on the sign of $E_{\mathrm{f}}-E$.

\section{Stiffness of a Nanosized Shell}

As in the case of porous materials thin films give us another example of structures with significant influence of surface effects. In this section we discuss the governing equations of the linear theory of plates and shells taking into account the surface properties. The main attention is paid here to the constitutive equations for the stress resultant and couple stress tensors. We consider the Reissner-type model of plates and shells. Within the framework of this theory a shell is assumed to be a deformable surface whose kinematics is described by the translation field $\mathbf{u}$ and by the rotation field $\vartheta$, see e.g. [24, 25, 27].

Equilibrium equations take the form

$$
\nabla_{S} \cdot \mathbf{T}+\mathbf{q}=\mathbf{0}, \quad \nabla_{S} \cdot \mathbf{M}+\mathbf{T}_{\times}+\mathbf{m}=\mathbf{0},
$$

where $\mathbf{T}$ and $\mathbf{M}$ are the stress resultant and stress couple tensors, respectively, $\mathbf{q}$ and $\mathbf{m}$ are the external surface loads and moments, and $\mathbf{T}_{\times}$denotes the vectorial invariant of the second-order tensor $\mathbf{T}$, see [27].

For the derivation of the constitutive equations for $\mathbf{T}$ and $\mathbf{M}$ we use the throughthe-thickness integration procedure of equilibrium Eqs. (1) and (2) in the case of a shell-like body, see Fig. 2. We assume that the shell-like body consists of three layers of thickness $h_{\mathrm{f}}, h_{\mathrm{c}}$, and $h_{\mathrm{f}}, E$ and $v$ are the Young modulus and Poisson ratio of the core, $h=h_{\mathrm{c}}+2 h_{\mathrm{f}}$ is the total thickness, $E_{\mathrm{f}}$ and $v_{\mathrm{f}}$ are the Young modulus and Poisson ratio of the surface layers, respectively. The surface stresses $\tau_{ \pm}$act only on the faces $\Omega_{ \pm}, \Omega_{3}=\Omega_{-} \cup \Omega_{+}$. In other words, we consider a three-layered shell-like body with surface stresses acting on its faces.

The through-the-thickness integration procedure is presented in many books and papers, see e.g. [27] and the reference in it. The procedure is extended to the case of surface stresses in $[7,8]$. As a result we obtain the relations

$$
\begin{gathered}
\mathbf{T}=\left\langle(\mathbf{A}-z \mathbf{B})^{-1} \cdot \boldsymbol{\sigma}\right\rangle+\boldsymbol{\tau}_{+}+\boldsymbol{\tau}_{-}, \quad\langle(\ldots)\rangle=\int_{-h / 2}^{h / 2}(\ldots) G d z, \\
\mathbf{M}=-\left\langle(\mathbf{A}-z \mathbf{B})^{-1} \cdot z \boldsymbol{\sigma} \times \mathbf{n}\right\rangle-\frac{h}{2}\left(\boldsymbol{\tau}_{+}-\boldsymbol{\tau}_{-}\right) \times \mathbf{n},
\end{gathered}
$$




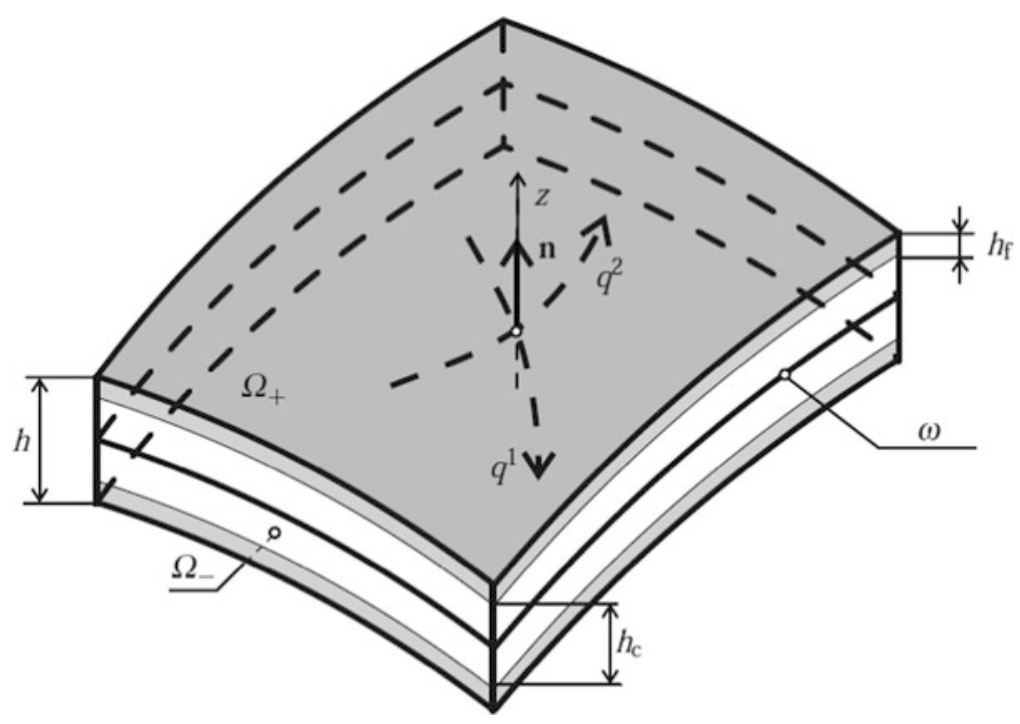

Fig. 2 Shell-like body

and

$$
\begin{aligned}
& \mathbf{q}=G_{+} \boldsymbol{\varphi}_{+}-G_{-} \boldsymbol{\varphi}_{-}, \quad \mathbf{m}=\frac{h}{2} G_{+} \mathbf{n} \times \boldsymbol{\varphi}_{+}+\frac{h}{2} G_{-} \mathbf{n} \times \boldsymbol{\varphi}_{-}, \\
& G=G(z) \equiv \operatorname{det}(\mathbf{A}-z \mathbf{B}), \quad G_{ \pm}=G( \pm h / 2),
\end{aligned}
$$

where $\mathbf{B}=-\nabla_{S} \mathbf{n}$ is the curvature tensor of the shell base surface $\omega, \mathbf{n}$ is the unit normal to $\omega$. Here and in what follows we assume that $h\|\mathbf{B}\| \ll 1$, i.e. we consider shallow thin shells. With this accuracy, the following equations can be also used

$$
\mathbf{T}=\langle\mathbf{A} \cdot \boldsymbol{\sigma}\rangle+\boldsymbol{\tau}_{+}+\boldsymbol{\tau}_{-}, \quad \mathbf{M}=-\langle\mathbf{A} \cdot z \boldsymbol{\sigma} \times \mathbf{n}\rangle-\frac{h}{2}\left(\boldsymbol{\tau}_{+}-\boldsymbol{\tau}_{-}\right) \times \mathbf{n} .
$$

For the derivation of $\mathbf{T}$ and $\mathbf{M}$ as the functions of the surface strain measures we use Eq. (17) with the approximation

$$
\mathbf{u}\left(q^{1}, q^{2}, z\right)=\mathbf{w}\left(q^{1}, q^{2}\right)-z \vartheta\left(q^{1}, q^{2}\right), \quad \mathbf{n} \cdot \vartheta=0 .
$$

This approximation is used in the theories of shear-deformable plates and shells, see e.g. [27], $\mathbf{w}$ is the translation vector of shell base surface $\omega, \boldsymbol{\vartheta}$ is the rotation vector of the shell cross-section, and $q^{1}, q^{2}$ are the convected coordinates on $\omega$.

Application of Eq. (18) to Eq. (17) leads to the following relations

$$
\mathbf{T}=C_{1} \mathbf{E}+C_{2} \mathbf{A t r} \mathbf{E}+\Gamma \boldsymbol{\gamma} \otimes \mathbf{n}, \quad \mathbf{M}=-\left[D_{1} \mathbf{K}+D_{2} \mathbf{A t r} \mathbf{K}\right] \times \mathbf{n},
$$


where $\mathbf{E}, \mathbf{K}$, and $\boldsymbol{\gamma}$ are the surface strain measures given by

$$
\begin{aligned}
& \mathbf{E}=\frac{1}{2}\left(\nabla_{S} \mathbf{w} \cdot \mathbf{A}+\mathbf{A} \cdot\left(\nabla_{S} \mathbf{w}\right)^{T}\right), \quad \mathbf{K}=\frac{1}{2}\left(\nabla_{S} \vartheta \cdot \mathbf{A}+\mathbf{A} \cdot\left(\nabla_{S} \vartheta\right)^{T}\right), \\
& \boldsymbol{\gamma}=\nabla_{S}(\mathbf{w} \cdot \mathbf{n})-\vartheta
\end{aligned}
$$

$C_{1}, C_{2}$ are the tangential stiffness parameters, $D_{1}$ and $D_{2}$ are the bending stiffness parameters, and $\Gamma$ is the transverse shear stiffness. The stiffness parameters are given by formulae

$$
\begin{aligned}
C_{1} & =2 C_{22}+4 \mu_{S}, \quad C_{2}=C_{11}-C_{22}+2 \lambda_{S}, \\
D_{1} & =2 D_{22}+h^{2} \mu_{S}, \quad D_{2}=D_{33}-D_{22}+\frac{h^{2}}{2} \lambda_{S}, \quad \Gamma=\ell^{2} D_{22}, \\
C_{11} & =\frac{1}{2}\left(\frac{2 E_{\mathrm{f}} h_{\mathrm{f}}}{1-v_{\mathrm{f}}}+\frac{E h_{\mathrm{c}}}{1-v}\right), \quad C_{22}=\frac{1}{2}\left(\frac{2 E_{\mathrm{f}} h_{\mathrm{f}}}{1+v_{\mathrm{f}}}+\frac{E h_{\mathrm{c}}}{1+v}\right), \\
D_{22} & =\frac{1}{24}\left[\frac{E_{\mathrm{f}}\left(h^{3}-h_{\mathrm{c}}^{3}\right)}{1+v_{\mathrm{f}}}+\frac{E h_{\mathrm{c}}^{3}}{1+v}\right], \quad D_{33}=\frac{1}{24}\left[\frac{E_{\mathrm{f}}\left(h^{3}-h_{\mathrm{c}}^{3}\right)}{1-v_{\mathrm{f}}}+\frac{E h_{\mathrm{c}}^{3}}{1-v}\right],
\end{aligned}
$$

where $\ell$ is the minimal positive root of the following equation

$$
\mu_{0} \cos \ell \frac{h_{\mathrm{f}}}{2} \cos \ell \frac{h_{\mathrm{c}}}{2}-\sin \ell \frac{h_{\mathrm{f}}}{2} \sin \ell \frac{h_{\mathrm{c}}}{2}=0, \quad \mu_{0}=\mu / \mu_{\mathrm{f}},
$$

$\mu$ and $\mu_{\mathrm{f}}$ are the shear moduli of the shell core and faces, respectively.

The effective tangential and bending stiffness take the form

$$
\begin{aligned}
C^{*} & \equiv C_{1}+C_{2}=\frac{2 E_{\mathrm{f}} h_{\mathrm{f}}}{1-v_{\mathrm{f}}^{2}}+\frac{E h_{\mathrm{c}}}{1-v^{2}}+4 \mu_{S}+2 \lambda_{S}, \\
D^{*} & \equiv D_{1}+D_{2}=\frac{1}{12}\left[\frac{E_{\mathrm{f}}\left(h^{3}-h_{\mathrm{c}}^{3}\right)}{1-v_{\mathrm{f}}^{2}}+\frac{E h_{\mathrm{c}}^{3}}{1-v^{2}}\right]+\frac{h^{2}}{2}\left(2 \mu_{S}+\lambda_{S}\right) .
\end{aligned}
$$

The stiffness parameters depend on the elastic moduli of the bulk material, i.e. on $E, \nu, E_{\mathrm{f}}$, and $\nu_{\mathrm{f}}$, on thicknesses $h_{\mathrm{c}}$ and $h_{\mathrm{f}}$, as well as on $2 \mu_{S}+\lambda_{S}$. Determination of the stiffness parameters $C_{11}, C_{22}, D_{22}$, and $D_{33}$ are discussed in [24, 25] in the case of three-layered and functionally graded plates. In the case of homogeneous bulk material, i.e. when $E_{\mathrm{f}}=E, v_{\mathrm{f}}=v$, these relations take the form as presented in $[7,8]$.

As in the case of the rod the surface elasticity leads to increasing of $C^{*}$ and $D^{*}$. This influence is significant if the shell thickness has the same order as the characteristic length parameter $d=\left(2 \mu_{S}+\lambda_{S}\right) / E$, i.e. when $h \sim d$. Note that $C^{*} \sim d$ and $D^{*} \sim h^{2} d$ when $h \rightarrow 0$, while in the classical plates and shells theory $C^{*} \sim h$ and $D^{*} \sim h^{3}$. As an example let us take the values used in $[1,18,26]$. 


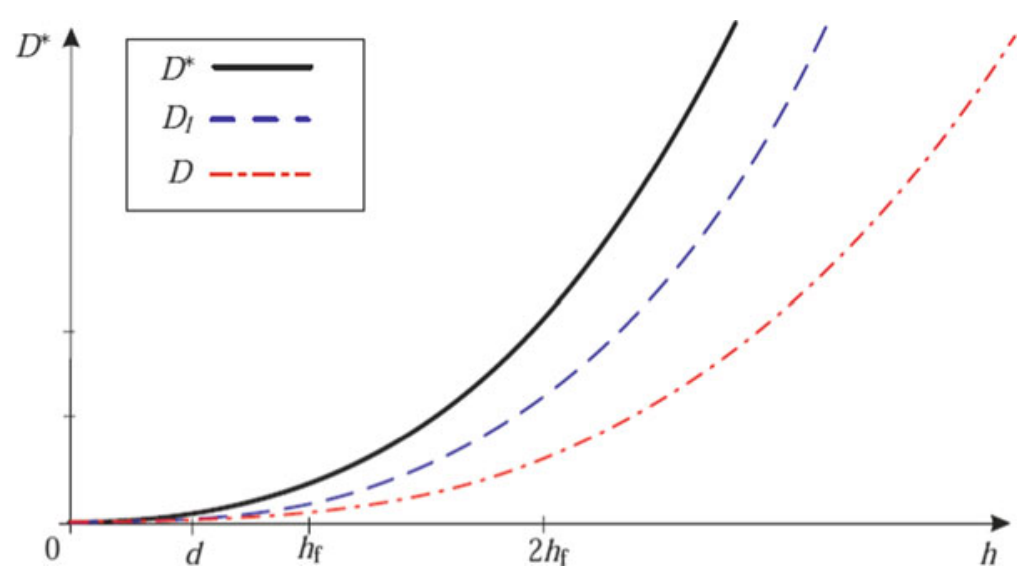

Fig. 3 Bending stiffness vs. $h$

We set $\mu=34.7 \mathrm{GPa}, v=0.3, \lambda_{S}=-3.48912 \mathrm{~N} / \mathrm{m}$, and $\mu_{S}=6.2178 \mathrm{~N} / \mathrm{m}$. In $[7,8]$ it is shown that for $h>50 \mathrm{~nm}$ the influence of surface stresses is negligible.

The change of the elastic properties of the bulk material near the shell faces can lead to increasing or decreasing values of $C^{*}$ and $D^{*}$. This depends on the sign of $E_{\mathrm{f}}-E$ and the Poisson ratios. The graphs of bending stiffness are given in Fig. 3 for small values of thickness. Here $D$ is the classical bending stiffness and $D_{l}$ is the bending stiffness of the three-layered plate [24, 25]

$$
D=\frac{E h^{3}}{12\left(1-v^{2}\right)}, \quad D_{l}=\frac{1}{12}\left[\frac{E_{\mathrm{f}}\left(h^{3}-h_{\mathrm{c}}^{3}\right)}{1-v_{\mathrm{f}}^{2}}+\frac{E h_{\mathrm{c}}^{3}}{1-v^{2}}\right]
$$

and is assumed to be $E_{\mathrm{f}}>E$.

\section{Conclusion}

We discuss the influence of surface effects on the effective properties of materials such as the effective bending stiffness of plates or the stiffness of rods. The interest to the investigation of the surface effects has recently grown with respect to nanomechanics. The surface effects play an important role for such nanosized materials as films, nanoporous materials, etc. We consider two models of surface effects. The first one is based on the concept of surface stresses which are the generalization of the surface tension for solids. The second one (more classical approach) is based on the consideration of the thin surface layer whose mechanical properties differ from the bulk material. Within the framework of these models we present the effective stiffness properties of plates, shells and nanoporous rods. 
The surface stresses have an influence on the effective stiffness properties of the elastic plates and shells. In the linear case this modification reduces to the addition of new terms to the elastic stiffness parameters. We show that the surface elasticity makes a shell stiffer in comparison with the shell without surface stresses. The second model shows that the effective stiffness of a plate with surface layers depends on the ratio of the elastic moduli of both the bulk and the surface materials.

Applying these two models we propose a model which takes into account not only the surface stresses but also the surface layer. This model predicts more complex behavior of the nanosized material. In particular, the nanoporous rod may be stiffer or softer then the solid rod and the effective stiffness may be a nonmonotonous function of the size of the voids.

Acknowledgements The second author was supported by the DFG grant No. AL 341/33-1 and by the RFBR with the grant No. 09-01-00459.

\section{References}

1. Duan, H.L., Wang, J.X., Karihaloo, B.L.: Theory of elasticity at the nanoscale. Adv. Appl. Mech. 42, 1-68 (2008). Elsevier

2. Gurtin, M.E., Murdoch, A.I.: A continuum theory of elastic material surfaces. Arch. Rat. Mech. Anal. 57(4), 291-323 (1975)

3. Orowan, E.: Surface energy and surface tension in solids and fluids. Proc. R. Soc. Lond. A 316(1527), 473-491 (1970)

4. Povstenko, Y.Z.: Theoretical investigation of phenomena caused by heterogeneous surface tension in solids. J. Mech. Phys. Sol. 41(9), 1499-1514 (1993)

5. Altenbach, H., Eremeyev, V.A., Lebedev, L.P.: On the existence of solution in the linear elasticity with surface stresses. ZAMM 90(7), 535-536 (2010)

6. Altenbach, H., Eremeyev, V.A., Lebedev, L.P.: On the spectrum and stiffness of an elastic body with surface stresses. ZAMM 91(9), 699-710 (2011). doi:10.1002/zamm.201000214

7. Altenbach, H., Eremeyev, V.A., Morozov, N.F.: Linear theory of shells taking into account surface stresses. Doklady Phys. 54(12), 531-535 (2009)

8. Altenbach, H., Eremeyev, V.A., Morozov, N.F.: On equations of the linear theory of shells with surface stresses taken into account. Mech. Solids 45(3), 331-342 (2010)

9. Dahmen, K., Lehwald, S., Ibach, H.: Bending of crystalline plates under the influence of surface stress - a finite element analysis. Surf. Sci. 446(1-2), 161-173 (2000)

10. Eremeyev, V.A., Altenbach, H., Morozov, N.F.: The influence of surface tension on the effective stiffness of nanosize plates. Doklady Phys. 54(2), 98-100 (2009)

11. Guo, J.G., Zhao, Y.P.: The size-dependent elastic properties of nanofilms with surface effects. J. Appl. Phys. 98(7), 074306 (2005) (11 pp)

12. Guo, J.G., Zhao, Y.P.: The size-dependent bending elastic properties of nanobeams with surface effects. Nanotechnology 18(29), 295701 (2007). 6 pp

13. Huang, D.W.: Size-dependent response of ultra-thin films with surface effects. Int. J. Sol. Struct. 45(2), 568-579 (2008)

14. Lu, P., He, L.H., Lee, H.P., Lu, C.: Thin plate theory including surface effects. Int. J. Sol. Struct. 43(16), 4631-4647 (2006)

15. Wang, Z.Q., Zhao, Y.P.: Self-instability and bending behaviors of nano plates. Acta Mech. Solida Sin. 22(6), 630-643 (2009) 
16. Wang, Z.Q., Zhao, Y.P., Huang, Z.P.: The effects of surface tension on the elastic properties of nano structures. Int. J. Eng. Sci. 48(2), 140-150 (2010)

17. Zhu, H.X., Wang, J.X., Karihaloo, B.: Effects of surface and initial stresses on the bending stiffness of trilayer plates and nanofilms. J. Mech. Mater. Struct. 4(3), 589-604 (2009)

18. Duan, H.L., Wang, J.X., Huang, Z.P., Karihaloo, B.L.: Size-dependent effective elastic constants of solids containing nano-inhomogeneities with interface stress. J. Mech. Phys. Sol. 53(7), 1574-1596 (2005)

19. Eremeyev, V.A., Morozov, N.F.: The effective stiffness of nanoporous rod. Doklady Phys. 55(6), 279-282 (2010)

20. Wang, Y., Weissmüller, J., Duan, H.L.: Mechanics of corrugated surfaces. J. Mech. Phys. Solids 58(10), 1552-1566 (2010)

21. Christensen, R.M.: Mechanics of Composite Materials. Dover, New York (2005)

22. Jones, R.M.: Mechanics of Composite Materials. Taylor \& Francis, Philadelphia (1999)

23. Schwarz, M. (ed.): Encyclopedia of Smart Materials, vol. 1, 2. Wiley, New York (2003)

24. Altenbach, H.: An alternative determination of transverse shear stiffnesses for sandwich and laminated plates. Int. J. Sol. Struct. 37(25), 3503-3520 (2000)

25. Altenbach, H., Eremeyev, V.A.: Direct approach based analysis of plates composed of functionally graded materials. Arch. Appl. Mech. 78(10), 775-794 (2008)

26. Wang, J.X., Duan, H.L., Huang, Z.P., Karihaloo, B.L.: A scaling law for properties of nanostructured materials. Proc. R. Soc. Lond. A 462(2069), 1355-1363 (2006)

27. Lebedev, L.P., Cloud, M.J., Eremeyev, V.A.: Tensor Analysis with Applications in Mechanics. World Scientific, New Jersey (2010) 